\title{
Effect of Roll-Bonding and Subsequent Annealing on Microstructure Evolution of Accumulative Roll Bonded Pure Copper
}

\author{
Yoji Miyajima $^{1,{ }^{*} 1,{ }^{* 2}}$, Midori Uchiyama ${ }^{1, * 3}$, Hiroki Adachi ${ }^{2}$, Toshiyuki Fujii ${ }^{3, * 4}$, Susumu Onaka ${ }^{1, * 2}$ \\ and Masaharu Kato ${ }^{1, * 5}$ \\ ${ }^{1}$ Department of Materials Science and Engineering, Tokyo Institute of Technology, Yokohama 226-8502, Japan \\ ${ }^{2}$ Department of Materials and Synchrotron Radiation Engineering, Graduate School of Engineering, University of Hyogo, \\ Himeji 671-2280, Japan \\ ${ }^{3}$ Department of Metallurgy and Ceramics Science, Tokyo Institute of Technology, Tokyo 152-8552, Japan
}

\begin{abstract}
The microstructure and texture evolution of an accumulative roll bonding (ARB) processed $4 \mathrm{~N}-\mathrm{Cu}$ with and without lubrication were compared in addition to the mechanical properties, in order to understand the effect of additional shear strain caused by the friction between the rolls and a sheet. Furthermore, subsequent annealing at $423 \mathrm{~K}, 448 \mathrm{~K}$ and $473 \mathrm{~K}$ was applied for $4 \mathrm{~N}-\mathrm{Cu}$ ARB processed with and without lubrication, and, softening was observed for all temperatures. The texture change due to the ARB process and subsequent annealing were discussed using $\{111\}$ pole figures and ODF maps. As a result, a $45^{\circ}$ rotated Cube texture around ND was formed by additional shear strain, whereas the typical fcc rolling texture was formed by ARB with lubrication. At higher ARB cycles of $4 \mathrm{~N}-\mathrm{Cu}$, discontinuous recrystallization occurs due to its medium stacking fault energy. Cube orientation appeared in annealed-ARB processed $4 \mathrm{~N}-\mathrm{Cu}$ with lubrication, whereas no specific texture appeared in annealed-ARB processed 4N-Cu without lubrication [doi:10.2320/matertrans.MH201506]
\end{abstract}

(Received February 29, 2016; Accepted May 13, 2016; Published June 24, 2016)

Keywords: severe plastic deformation (SPD), accumulative roll bonding (ARB), texture, copper, electron back scattering diffraction (EBSD)

\section{Introduction}

An accumulative roll bonding (ARB) process is known as one of the severe plastic deformation (SPD) processes which can give much larger equivalent strain than that of normal plastic deformation, and generally, the equivalent strain becomes larger than four in the SPD processes ${ }^{1)}$. The ARB process contains a roll bonding with the rolling reduction of $50 \%$, and therefore, the microstructure evolution of the ARB processed metals is expected to be similar with that of rolled metals.

There are essentially two types of surface condition of rolls for the ARB process, i.e. with and without lubrication. The difference between with and without lubrication is the presence of machine oil on the surface of the rolls, which gives different friction between the surfaces of the rolls and metal plates. If the surface friction is lowered by the machine oil, the deformation of the one cycle of the ARB process can be treated as plane strain compression, of which equivalent strain is about $0.8^{2}$. On the other hand, the larger friction gives larger strain adjacent to the surface, and therefore enhances the microstructural evolution close to the surface, which is described as the additional shear strain ${ }^{3-5}$. Furthermore, the additional shear strain was reproduced in finite element method (FEM) ${ }^{6}$.

It is also well known that the application of a same mode of deformation on polycrystalline metals results in creating a

\footnotetext{
${ }^{* 1}$ Corresponding author, E-mail: miyajima.y.ab@m.titech.ac.jp

${ }^{*}$ Present address: School of Materials and Chemical Technology, Tokyo Institute of Technology, Yokohama 226-8502, Japan

${ }^{* 3}$ Graduate Student, Tokyo Institute of Technology. Present address: IHI Corporation, Yokohama 235-8501, Japan

${ }^{* 4}$ Present address: School of Materials and Chemical Technology, Tokyo Institute of Technology, Tokyo 152-8552, Japan

${ }^{*}$ Present address: Nippon Steel \& Sumitomo Metal Corporation, Futtsu 293-8511, Japan
}

strong deformation texture. For instance, rolling deformation on face centred cubic (FCC) metals produces strong rolling texture which consists of some components; Brass $\{110\}<112>, S\{123\}<634>$, and Copper $\{110\}<112>$ orientations ${ }^{7,8)}$. Since it is essentially impossible to apply the "in-situ" microstructural observations/measurements during SPD processes, the texture analysis becomes a strong tool in order to understand the microstructural evolution during the SPD process. Although there are texture analysis on the ARB processed metals ${ }^{3-5,9-15)}$, especially, there are not many reports on $\mathrm{Cu}^{16)}$. Thus, we studied the texture evolution of pure $\mathrm{Cu}$ during $\mathrm{ARB}$ process with and without lubrication.

\section{Experimental}

The starting materials for the ARB process were fully recrystallized $99.99 \%$ purity $\mathrm{Cu}$ sheets having the mean grain size of $32 \mu \mathrm{m}$ as a result of the annealing at $873 \mathrm{~K}$ for $7.2 \mathrm{ks}$. The thickness, width and length of the sheets were $1 \mathrm{~mm}$, $90 \mathrm{~mm}$ and $250 \mathrm{~mm}$, respectively. The ARB process was performed up to eight cycles with and without lubrication at room temperature. Hereafter, the $N$ th $\mathrm{ARB}$ processed $\mathrm{Cu}$ sheet is denoted as ARB $N \mathrm{c}$, and here, $N$ is the ARB cycle number. It should be noted that annealed $4 \mathrm{~N}-\mathrm{Cu}$ used as the starting material is treated as ARB 0c.

The microstructure of the ARB processed $\mathrm{Cu}$ was investigated by EBSD analysis in a field emission type scanning electron microscope (FE-SEM), JEOL JSM-6500FK, operated at $15 \mathrm{kV}$. The EBSD analysis was carried out using a program, TSL OIM data collection 4.6. The specimens for the EBSD observation were cut using an arc discharge wire cutting machine, and then, the damaged surface layer was removed by mechanical polishing with $\mathrm{SiC}$ papers. Finally, electrolytic polishing was performed in a solution of phosphoric acid, ethanol and water of 1:1:2 ratio in volume. The sample coordinates are defined by rolling direction (RD), 


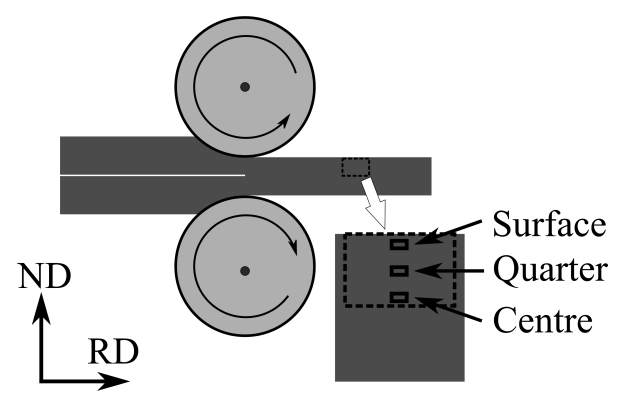

Fig. 1 A schematic illustration of the ARB process and the definition of measured area along ND; surface, quarter and centre.

transverse direction (TD) and normal direction (ND). The microstructure observations were performed from TD. As shown in Fig. 1, the observation areas were three along ND; close to the surface (surface), a quarter thickness distance from the surface (quarter) and close to the centre (centre), in the case of the ARB without lubrication. Whereas, the observation area is only quarter for the ARB with lubrication, since it was reported that the ARB with lubrication produces almost homogeneous structure along ND in the case of $\mathrm{Al}^{13)}$.

From EBSD data, two types of grain boundary (GB) were defined; one is low angle grain boundary (LAGB) and the other is high angle grain boundary (HAGB). Due to the limitation of the measurement, GB having misorientation angle $\theta$ less than $2^{\circ}$ was ignored. Thus, LAGB and HAGB were $2^{\circ} \leq$ $\theta<15^{\circ}$ and $15^{\circ} \leq \theta$, respectively. It should be noted that the $\{111\}$ pole figures and orientation distribution function (ODF) maps were constructed from EBSD data.

As the mechanical test, the uniaxial tensile tests with the gage length of $10 \mathrm{~mm}$, thickness of $1 \mathrm{~mm}$ and width of $3 \mathrm{~mm}$, and, the Vickers hardness tests on the plane normal to TD (TD plane) were performed. The Vickers hardness tests were carried out on three areas the same as EBSD measurements; surface, quarter and centre for without lubrication, and, only quarter for with lubrication.

Furthermore, ARB 6c with and without lubrication were subjected to annealing inside an oil bath with the temperatures of $423 \mathrm{~K}, 448 \mathrm{~K}$ and $473 \mathrm{~K}$. The change in Vickers hardness depending on annealing time was measured, and, EBSD measurements were performed for the texture analysis after the softening due to annealing stopped.

\section{Results and Discussion}

\subsection{Effect of roll-bonding condition on ARB processed $4 \mathrm{~N}-\mathrm{Cu}$}

From EBSD data, GB and HAGB maps were constructed. The former displays both LAGB and HAGB, whereas, the latter displays only HAGB. From these maps, mean separation of GB and HAGB along ND ( $d_{\mathrm{GB}}^{\mathrm{ND}}$ and $\left.d_{\mathrm{HAGB}}^{\mathrm{ND}}\right)$ were evaluated using interception method as shown in Fig. 2. The initial grain size is about $32 \mu \mathrm{m}$ both for $d_{\mathrm{GB}}^{\mathrm{ND}}$ and $d_{\mathrm{HAGB}}^{\mathrm{ND}}$. Both $\mathrm{ARB}$ with and without lubrication reduces $d_{\mathrm{GB}}^{\mathrm{ND}}$ and $d_{\mathrm{HAGB}}^{\mathrm{ND}}$ with increasing $N$, and, $d_{\mathrm{GB}}^{\mathrm{ND}}$ is always smaller than $d_{\mathrm{HAGB}}^{\mathrm{ND}}$. However, the decrease in $d_{\mathrm{GB}}^{\mathrm{ND}}$ and $d_{\mathrm{HAGB}}^{\mathrm{ND}}$ of the ARB with lubrication is smaller than these of the ARB without lubrication, which is associated with the grain refinement due to the

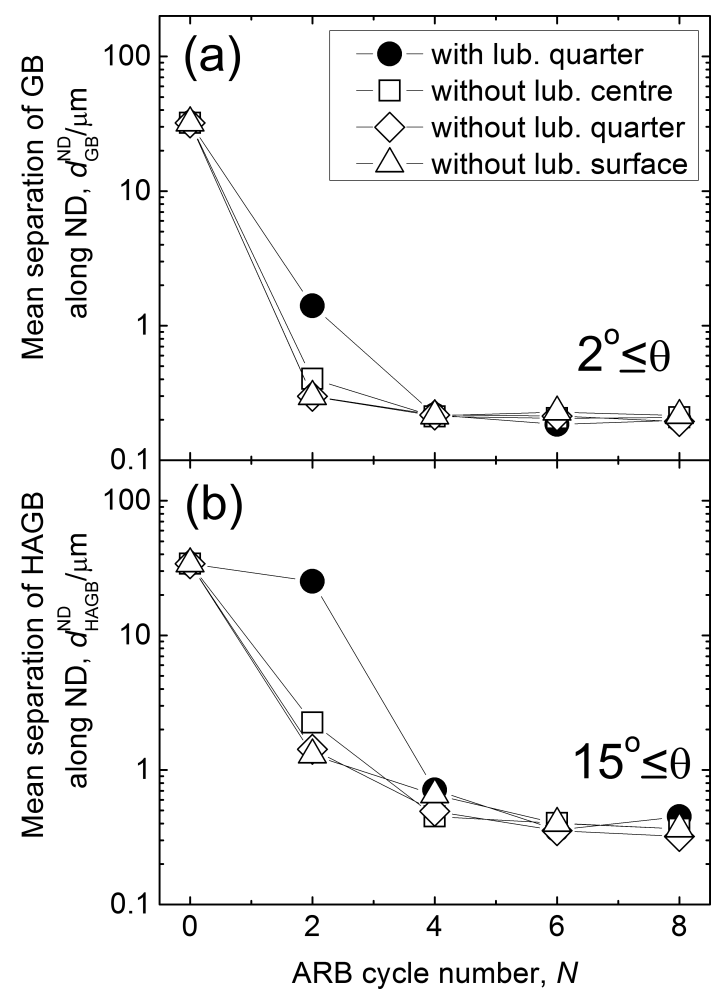

Fig. 2 Mean separation of (a) GB $\left(2^{\circ} \leq \theta\right)$ and (b) HAGB $\left(15^{\circ} \leq \theta\right)$ along ND. In the case of the ARB without lubrication, three areas displayed in Fig. 1 were measured.

additional shear strain caused by the friction between the surfaces of the sheets and roll ${ }^{3-5}$ ). The inhomogeneity along ND due to the ARB without lubrication can be strongly seen only at-2c for both $d_{\mathrm{GB}}^{\mathrm{ND}}$ and $d_{\mathrm{HAGB}}^{\mathrm{ND}}$ in Fig. 2. For instance, $d_{\mathrm{GB}}^{\mathrm{ND}}$ of ARB $2 \mathrm{c}$ at centre, quarter and surface are about $0.40 \mu \mathrm{m}$, $0.30 \mu \mathrm{m}$ and $0.30 \mu \mathrm{m}$, respectively. Whereas, $d_{\mathrm{HAGB}}^{\mathrm{ND}}$ of ARB $2 \mathrm{c}$ at centre, quarter and surface are about $2.3 \mu \mathrm{m}, 1.4 \mu \mathrm{m}$ and $1.3 \mu \mathrm{m}$, respectively. Obviously, the grain refinements of the area at centre is delayed compared with the areas at quarter and surface, and the inhomogeneity along ND can be seen. After $2 \mathrm{c}$, the more grain refined surface region is folded into the centre of the sheet due to the stacking/roll bonding during the ARB process, and, the grain size inhomogeneity along ND is reduced. Both $d_{\mathrm{GB}}^{\mathrm{ND}}$ and $d_{\mathrm{HAGB}}^{\mathrm{ND}}$ become almost constant after $4 \mathrm{c}$ and $6 \mathrm{c}$, respectively. It is known as the saturation of grain refinement when the SPD processes are used.

In the case of $d_{\mathrm{GB}}^{\mathrm{ND}}$ of $\mathrm{ARB} 8 \mathrm{c}$ without lubrication at centre, quarter and surface are almost $0.21 \mu \mathrm{m}, 0.19 \mu \mathrm{m}$ and $0.22 \mu \mathrm{m}$, respectively. Whereas, $d_{\mathrm{HAGB}}^{\mathrm{ND}}$ of ARB $8 \mathrm{c}$ without lubrication at centre, quarter and surface are about $0.37 \mu \mathrm{m}, 0.32 \mu \mathrm{m}$ and $0.37 \mu \mathrm{m}$, respectively. On the other hand, $d_{\mathrm{GB}}^{\mathrm{ND}}$ and $d_{\mathrm{HAGB}}^{\mathrm{ND}}$ of ARB 8c with lubrication are $0.20 \mu \mathrm{m}$ and $0.45 \mu \mathrm{m}$, respectively. In the case of $d_{\mathrm{HAGB}}^{\mathrm{ND}}$ of ARB 8c, the difference between with and without lubrication is relatively larger than the case of $d_{\mathrm{GB}}^{\mathrm{ND}}$

Figure 3 shows the Vickers hardness $h$ of ARB processed $4 \mathrm{~N}-\mathrm{Cu}$ with and without lubrication on the TD plane, and, the error bars show the standard deviation. Nevertheless, the sizes of the error bars are similar size of symbols in Fig. 3. Initially, $h$ is about $50 \mathrm{Hv}$ for ARB 0c. Then, $h$ drastically increases to about $140 \mathrm{Hv}$ at surface of the ARB 1c without lu- 


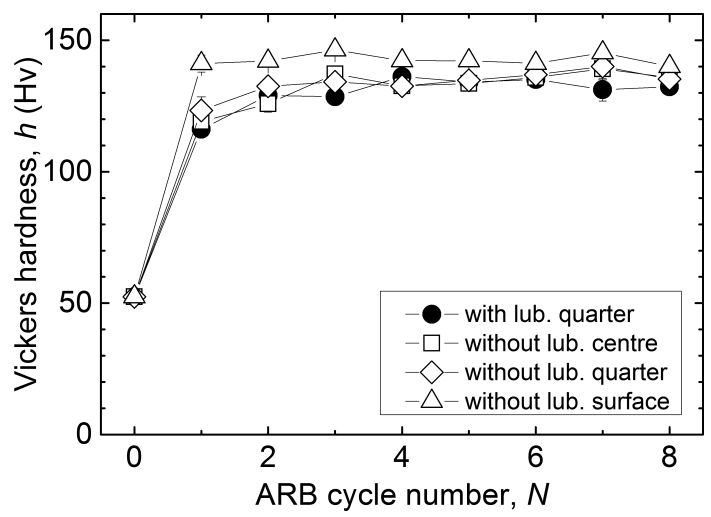

Fig. 3 Vickers hardness of ARB processed 4N-Cu with and without lubrication. In the case of the ARB without lubrication, three areas displayed in Fig. 1 were measured.

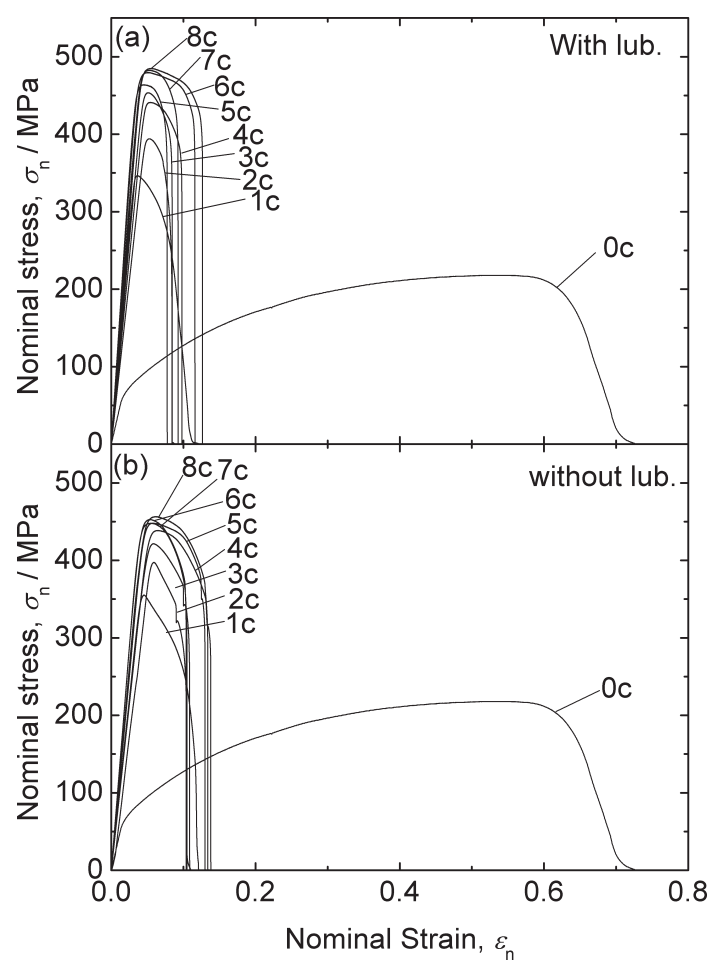

Fig. 4 Nominal stress versus nominal strain curves of ARB processed $4 \mathrm{~N}$ $\mathrm{Cu}$ (a) with and (b) without lubrication.

brication and the hardness of the other samples after 1c reaches to about $120 \mathrm{Hv}$. The hardness at the surface of ARB sample without lubrication seems to be constant after 1c, whereas the other increases gradually with increasing $N$ up to 4 , and then, saturate. The reason why the hardness at surface is always higher than those of the others is again associated with the additional grain refinement caused by the friction between the surfaces of sheet and rolls.

Figure 4 shows the nominal stress versus nominal strain curves of ARB processed 4N-Cu (a) with and (b) without lubrication. As can be seen, strength seems to increase with increasing $N$, but, it saturates after $6 \mathrm{c}$ and $5 \mathrm{c}$ for with and without lubrication, respectively. The ductility of ARB processed $4 \mathrm{~N}-\mathrm{Cu}$-sheets is much less than that for annealed $4 \mathrm{~N}-\mathrm{Cu}$.

From the s-s curves, $0.2 \%$ proof stress $\sigma_{0.2}$ and ultimate tensile stress (UTS) $\sigma_{\mathrm{UTS}}$ were evaluated and displayed in

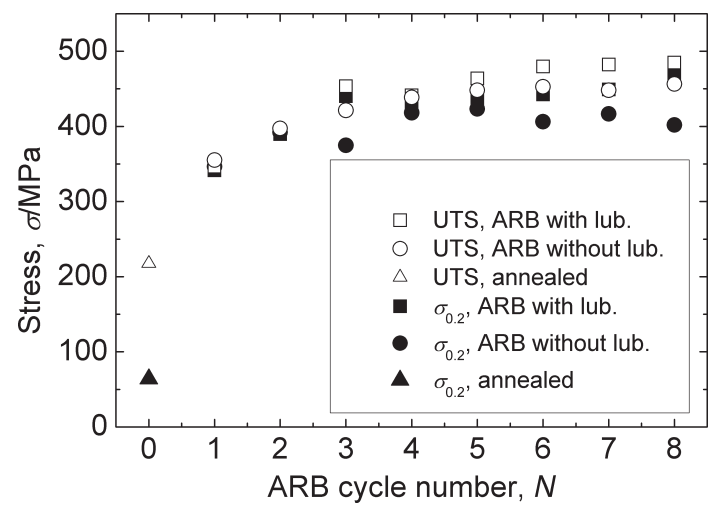

Fig. 5 UTS and $0.2 \%$ proof stress of ARB processed $4 \mathrm{~N}-\mathrm{Cu}$ with and without lubrication.

Fig. 5. The initial values of $\sigma_{0.2}$ and $\sigma_{\text {UTS }}$ are about $60 \mathrm{MPa}$ and $210 \mathrm{MPa}$ at $0 \mathrm{c}$, respectively. These values for both ARB with and without lubrication drastically increased to about $350 \mathrm{MPa}$ at $1 \mathrm{c}$. Then, those values become about $400 \mathrm{MPa}$ at 2c. Although $\sigma_{0.2}$ of the sample processed without lubrication seems to be independent of $N$, that of the sample processed with lubrication increases to about $450 \mathrm{MPa}$ at $3 \mathrm{c}$ and then saturate after $4 \mathrm{c}$. Up to $6 \mathrm{c}, \sigma_{\mathrm{UTS}}$ of the ARB with and without lubrication still gradually increases to $480 \mathrm{MPa}$ and $450 \mathrm{MPa}$, respectively, and then, saturate. The trend of $\sigma_{0.2}$ and $\sigma_{\mathrm{UTS}}$ is opposite compared with that of Vickers hardness, which will be discussed later.

The $\{111\}$ pole figures of ARB processed $4 \mathrm{~N}-\mathrm{Cu}$ with and without lubrication are shown in Fig. 6. As can be seen in Fig. 6(q), ARB 0c has Cube $\{001\}<100>$ texture which is known as rolling and recrystallization texture for fcc metals. There is no specific poles related to the typical fcc rolling texture at $2 \mathrm{c}$ since they are still transition state for both ARB with and without lubrication. In the case of the ARB $2 \mathrm{c}$ with lubrication, the poles move to the typical rolling texture such as Copper $\{112\}<111>$, Brass $\{110\}<112>$ and $S\{123\}<634>$ with increasing $N$. Whereas the ARB 2c without lubrication shows different texture at centre, quarter and surface. The texture of the ARB without lubrication at all locations are relatively similar up to $4 \mathrm{c}$. Then, that at centre and quarter still show relatively similar texture compared with the ARB with lubrication, but, that at surface shows different from the typical rolling texture which seems rather $45^{\circ}$ rotated Cube $\{001\}<110>$ texture around ND. It should be noted that this rotated Cube texture is also found in ARB processed commercial purity $\mathrm{Al}$ and $\mathrm{Ni}$ without lubrication ${ }^{3-5,9,14)}$. The difference is also associated with the additional shear strain caused by the surface friction due to the surfaces between the sheet and the rolls. The effect of additional shear strain is more prominent at surface region than that inside the sheets. The effect of additional shear strain still seems to exist close to the centre since the centre was the surfaces of the sheet before the last roll bonding. However, the texture at centre seems to be not exactly the same as that at surface.

Since pole figure is the stereographic projection of orientation on plane, sometimes it is difficult to distinguish some orientations. Thus, ODF maps are useful since they are sections in Euler angle space. In this study, Bunge's notation is used to represent Euler angle $\left(\phi_{1}, \Phi, \phi_{2}\right)$, such as, Copper 

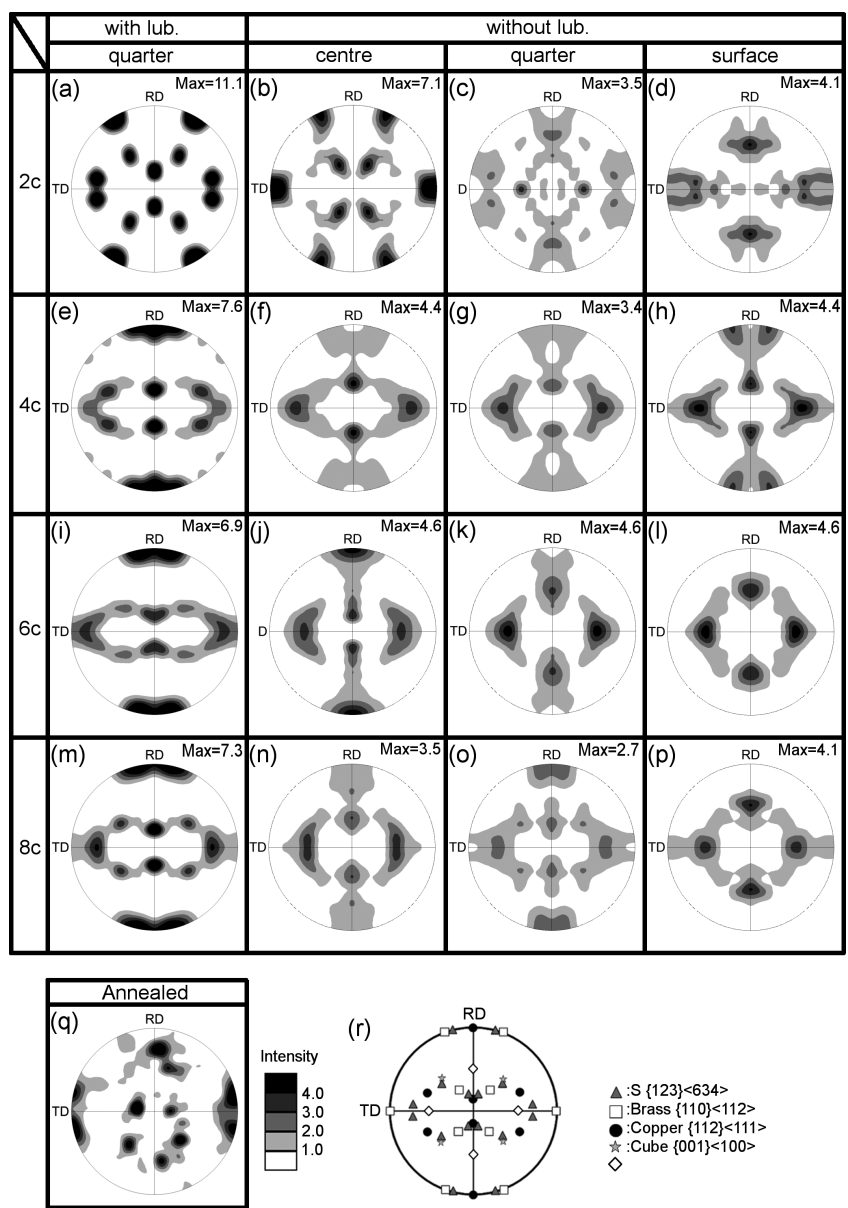

$\Delta: S\{123\}<634>$ $\square:$ Brass $\{110\}<112>$ : : Copper $\{112\}<111>$

Fig. $6\{111\}$ pole figures of ARB processed 4N-Cu: (a)-(d) are ARB 2c, (e)-(h) are ARB 4c, (i)-(1) are ARB 6c, and (m)-(p) are ARB 8c, respectively. The first column from the left is the results at quarter of ARB with lubrication. The second, third, and the fourth columns from the left are at centre, quarter, and surface of ARB without lubrication, respectively. (q) $\{111\}$ pole figure of annealed (ARB $0 \mathrm{c}$ ) $4 \mathrm{~N}-\mathrm{Cu}$, and (r) ideal orientations of the rolling texture along $\beta$ fibre, Cube and rotated Cube orientations.

$\left(90^{\circ}, 35^{\circ}, 45^{\circ}\right)$, Brass $\left(35^{\circ}, 45^{\circ}, 0^{\circ}\right), \mathrm{S}\left(59^{\circ}, 37^{\circ}, 63^{\circ}\right)$, Cube $\left(0^{\circ}\right.$, $\left.0^{\circ}, 0^{\circ}\right)$ and rotated cube $\left(45^{\circ}, 0^{\circ}, 0^{\circ}\right)$.

Figures 7 and 8 show the ODF maps of ARB processed $4 \mathrm{~N}-\mathrm{Cu}$ with and without lubrication on $\phi_{1}=0^{\circ}$ and $\phi_{2}=45^{\circ}$ sections, respectively. From the most left column shows the ODF maps of the ARB with lubrication, and, from the second most left column to the most right column show those of the ARB without lubrication at centre, quarter and surface, respectively. From the top to the bottom rows, ARB 2c, ARB $4 \mathrm{c}, \mathrm{ARB} 6 \mathrm{c}$ and ARB $8 \mathrm{c}$ are shown.

From Fig. 7 (d), (h), (l) and (p), it is confirmed that the surface of the ARB processed $4 \mathrm{~N}-\mathrm{Cu}$ without lubrication has strong texture consisting of $45^{\circ}$ rotated Cube around $\mathrm{ND}\left(45^{\circ}\right.$, $\left.0^{\circ}, 0^{\circ}\right)$. The intensity of the rotated Cube at surface of ARB without lubrication increases with increasing $N$ up to 6 , and then, slightly decreases after $8 \mathrm{c}$. Furthermore, rotated Cube can be found all the regions when $N=4,6$ and 8 . The strength of poles is listed from the stronger, surface, quarter and centre.

From Fig. 8 (e), (i) and (m), it is also confirmed that the typical rolling texture, such as Copper and Brass, exists after $4 \mathrm{c}$ with lubrication. Strictly speaking, Brass seems to appear only at $6 c$, and, Copper seems to be the main component but
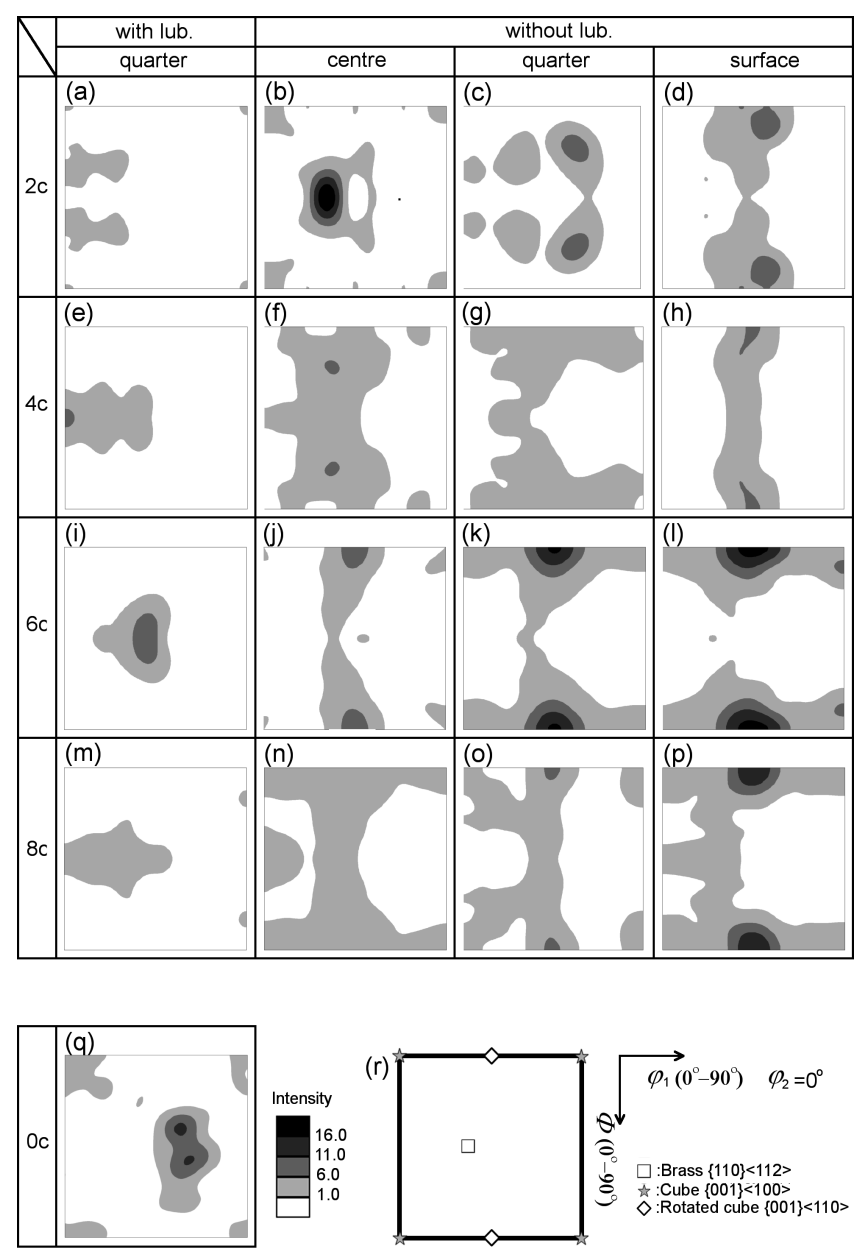

Fig. 7 ODF map on $\phi_{2}=0^{\circ}$ section of ARB processed $4 \mathrm{~N}-\mathrm{Cu}$ : (a)-(d) are ARB 2c, (e)-(h) are ARB 4c, (i)-(1) are ARB 6c, and (m)-(p) are ARB 8c, respectively. The first column from the left is the results at quarter of ARB with lubrication. The second, third, and the fourth columns from the left are at centre, quarter, and surface of ARB without lubrication, respectively. (q) annealed (ARB Oc) $4 \mathrm{~N}-\mathrm{Cu}$, and (r) ideal orientations on $\phi_{2}=0^{\circ}$ section.

the intensity is the highest at 4c. After 4c, intensity of Copper seems to decreases with increasing $N$. Weak poles of rolling texture components, such as Brass at $2 \mathrm{c}$ and Copper at $4 \mathrm{c}, 6 \mathrm{c}$ and $8 \mathrm{c}$, are also observed at the centre of ARB without lubrication.

Heason and Prangnell showed the texture evolution of A1100 aluminium alloy processed by ARB without lubrication, and, found out similar texture evolution compared with this study ${ }^{9)}$. They also qualitatively explained by using simulation that rotated Cube shift toward Copper orientation due to pure plane strain compression, whereas, Copper, Brass and $\mathrm{S}$ orientations shift toward rotated Cube due to the additional shear strain. The simulation results agree with the texture evolution observed in this study, if we consider that the ARB with lubrication and the centre region in the ARB without lubrication deform in plane strain compression, while an additional shear strain is applied to the surface and quarter regions in the ARB without lubrication. Therefore, the texture appeared in ARB processed $\mathrm{Cu}$ with and without lubrication can be understood qualitatively, apart from at ARB 8c, the strength of texture decreases compared with ARB 6c. If the same deformation is continuously applied, the texture evolu- 

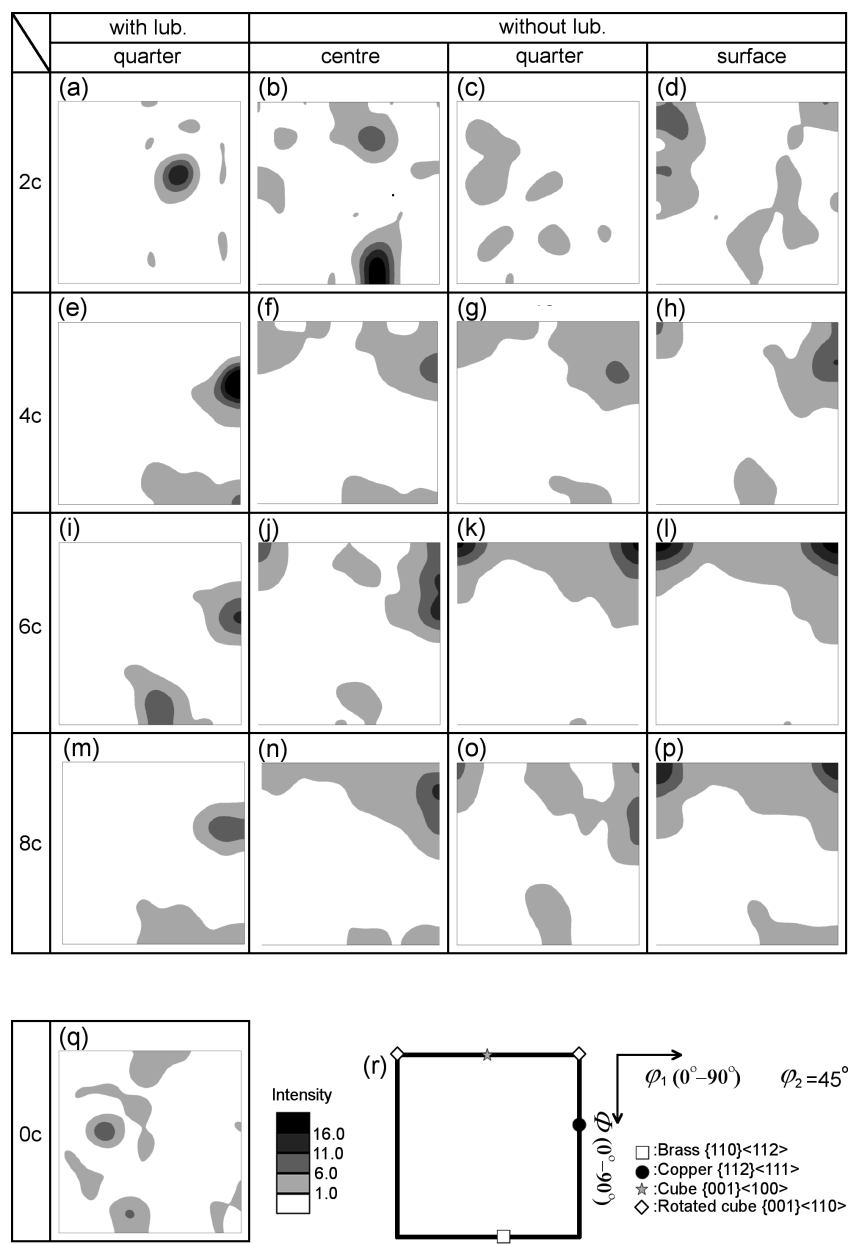

Fig. 8 ODF map on $\phi_{2}=45^{\circ}$ section of ARB processed $4 \mathrm{~N}-\mathrm{Cu}$ : (a)-(d) are ARB 2c, (e)-(h) are ARB 4c, (i)-(l) are ARB 6c, and (m)-(p) are ARB 8c, respectively. The first column from the left is the results at quarter of ARB with lubrication. The second, third, and the fourth columns from the left are at centre, quarter, and surface of ARB without lubrication, respectively. (q) annealed (ARB 0c) $4 \mathrm{~N}-\mathrm{Cu}$, and (r) ideal orientations on $\phi_{2}=45^{\circ}$ section.

tion should continue and intensity of poles is expected to be stronger.

The reason why rolling texture component in ARB with lubrication becomes weaker after $6 \mathrm{c}$ is associated with the grain growth during ARB process. From Fig. 2(b), $d_{\mathrm{HAGB}}^{\mathrm{ND}}$ of the ARB 8c with lubrication is slightly higher than that of the ARB 6c. This implies that grain growth may occur in ARB with lubrication at relatively higher cycles. In such case, if the new grains with different orientations from Copper texture appear, the decrease of the intensity can be understood. Although no significant increase of the grain size can be seen in Fig. 2 in the case of ARB without lubrication, new grains having different orientations may still appear. If so, the decrease of the intensity of rotated Cube can also be understood. The grain growth during ARB process has also been observed in $4 \mathrm{~N}-\mathrm{Al}$ case, though the decrease of intensity for the rolling texture components was not observed ${ }^{15)}$. In the Al case, the intensity of rolling texture component rather increased with increasing $N$.

The different texture evolution between $\mathrm{Cu}$ and $\mathrm{Al}$ is attributed to the difference in stacking fault energy (SFE). It is known that UFG metals having high SFE shows continuous

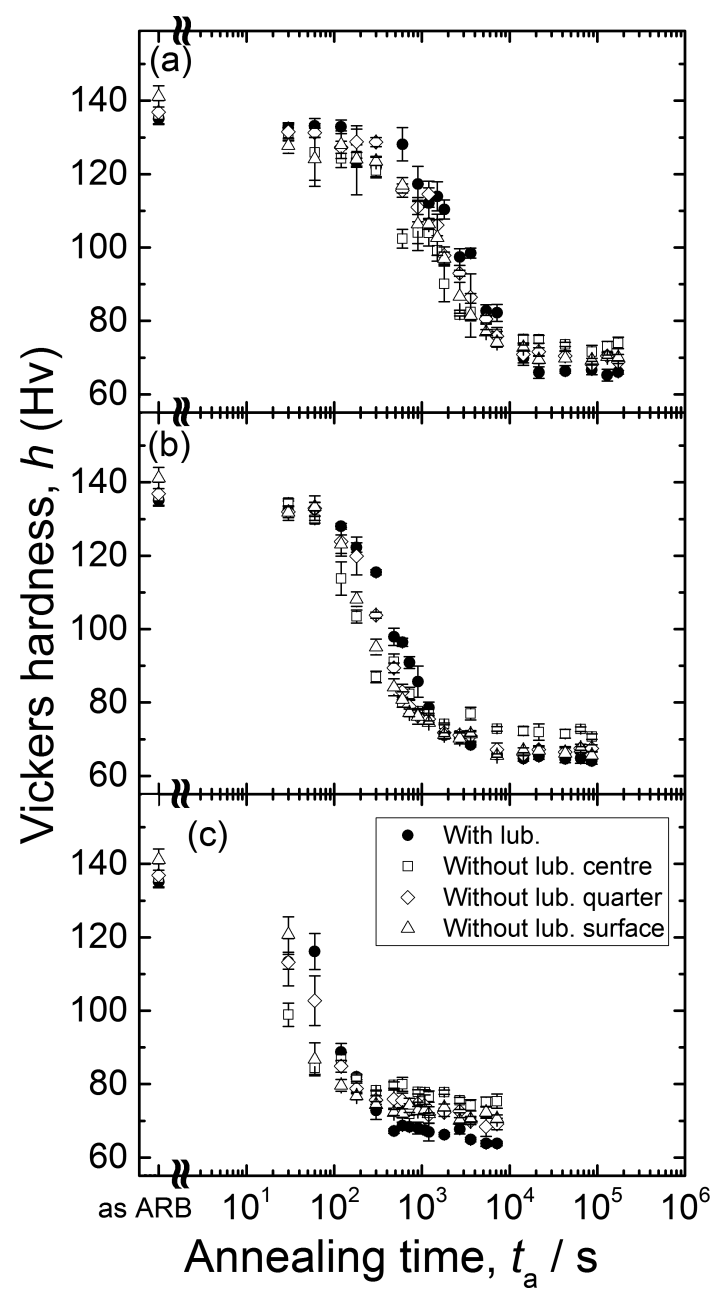

Fig. 9 Annealing time dependence on Vickers hardness of ARB 6c with and without lubrication of which annealing temperature at (a) $423 \mathrm{~K}$, (b) $448 \mathrm{~K}$ and (c) $473 \mathrm{~K}$.

recrystallization (recovery type), whereas, those having midium-low SFE shows discontinuous recrystallization (recrystallization type ${ }^{17)}$. When the continuous recrystallization occurs, strong change in texture is not observed since some pre-existing grains increase their size. On the other hand, when the discontinuous recrystallization occurs, new grains with different orientations from pre-existing grains appear. It is noted that $\mathrm{Cu}$ and $\mathrm{Ni}$ are classified to be medium SFE fcc metals, whereas, $\mathrm{Al}$ is classified to be high SFE fcc metal.

Thus, the intensity of fcc rolling texture components in ARB processed $4 \mathrm{~N}-\mathrm{Cu}$ with lubrication and rotated Cube in ARB processed $4 \mathrm{~N}-\mathrm{Cu}$ without lubrication decrease at relatively high $N$, since dynamic discontinuous recrystallization occurs due to the medium SFE. On the other hand, 4N-Al ARB processed with lubrication is thought to show dynamic continuous recrystallization, and therefore, the intensity for the rolling texture components increases with increasing $N$.

The effect of the different texture evolution between $4 \mathrm{~N}$ $\mathrm{Cu}$ ARB processed with and without lubrication affects the mechanical properties, such as the saturation value of $\sigma_{0.2}$ and $\sigma_{\text {UTS }}$. Because, the difference of the values of $\sigma_{0.2}$ and $\sigma_{\text {UTS }}$ cannot be simply associated with the difference of the grain size, such as $d_{\mathrm{GB}}^{\mathrm{ND}}$ and $d_{\mathrm{HAGB}}^{\mathrm{ND}}$. For instance, $d_{\mathrm{GB}}^{\mathrm{ND}}$ of $\mathrm{ARB}$ $8 \mathrm{c}$ with and without lubrication are almost constant values, 

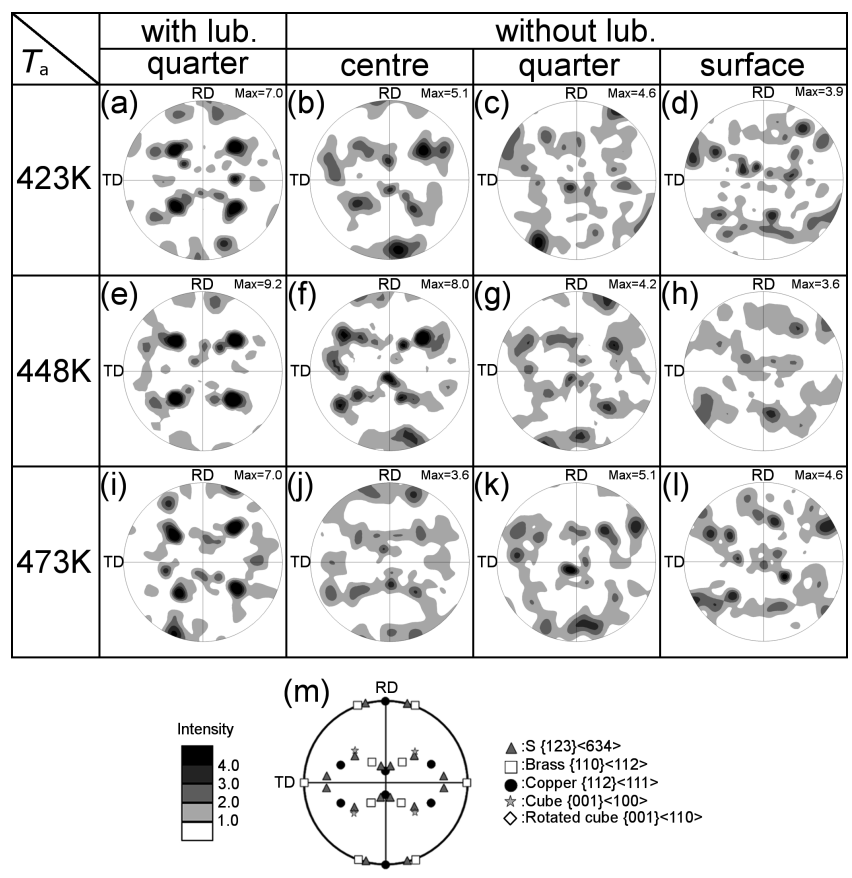

Fig. $10\{111\}$ pole figures of ARB processed 4N-Cu: (a)-(d) are ARB 6c annealed at $423 \mathrm{~K},(\mathrm{e})-(\mathrm{h})$ are ARB $6 \mathrm{c}$ annealed at $448 \mathrm{~K}$ and (i)-(1) are ARB $6 \mathrm{c}$ annealed at $473 \mathrm{~K}$. The first column from the left is the results at quarter of ARB with lubrication. The second, third, and the fourth columns from the left are at centre, quarter, and surface of ARB without lubrication, respectively. (m) ideal orientations of the rolling texture along $\beta$ fibre, Cube and rotated Cube orientations.

and $d_{\mathrm{HAGB}}^{\mathrm{ND}}$ of $\mathrm{ARB}$ with lubrication is slightly smaller than that of without lubrication. This is again the opposite trend if only grain refinement strengthening is considered. However, it is known that the mechanical properties of material having strong texture can be isotropic since some mechanical properties can be anisotropic such as yield stress. Thus, the ARB processed $4 \mathrm{~N}-\mathrm{Cu}$ with and without lubrication may show the difference in yield stress due to their strong and different texture.

\subsection{Effect of annealing on ARB processed $4 \mathrm{~N}-\mathrm{Cu}$}

Figure 9 shows the changes in Vickers hardness of ARB $6 \mathrm{c}$ with and without lubrication due to annealing at $423 \mathrm{~K}, 448 \mathrm{~K}$ and $473 \mathrm{~K}$. The maximum and the minimum error bars express the standard deviation. In the case of the annealing temperature $T_{a}$ at $423 \mathrm{~K}, h$ decreases from about $140 \mathrm{Hv}$ for the initial value of the ARB 6c down to $130 \mathrm{Hv}$ for annealing time $t_{a}$ at about $300 \mathrm{~s}$. Then, $h$ rapidly decreases down to about $70 \mathrm{Hv}$ for $t_{a}$ at about $14 \mathrm{ks}$ and saturate after about $14 \mathrm{ks}$. In the case of $T_{a}=448 \mathrm{~K}, h$ decreases down to about $130 \mathrm{Hv}$ when $t_{a} \approx 60 \mathrm{~s}$, and, rapidly decreases down to about $70 \mathrm{Hv}$ when $t_{a} \approx 7.2 \mathrm{ks}$. In the case of $T_{a}=473 \mathrm{~K}, h$ decreases immediately from about $140 \mathrm{Hv}$ down to about $70 \mathrm{Hv}$ when $t_{a} \approx 500 \mathrm{~s}$, and then, saturates. When lining up in order of decreasing $h$ at the saturation region: ARB without lubrication at centre, that at quarter, that at surface and with lubrication.

EBSD measurements were performed with samples of which $h$ reaches the saturation region, and, $\{111\}$ pole figures and ODF maps on $\phi_{2}=0^{\circ}$ section of annealed ARB $6 \mathrm{c}$ are displayed in Figs. 10 and 11, respectively. Top, medium and

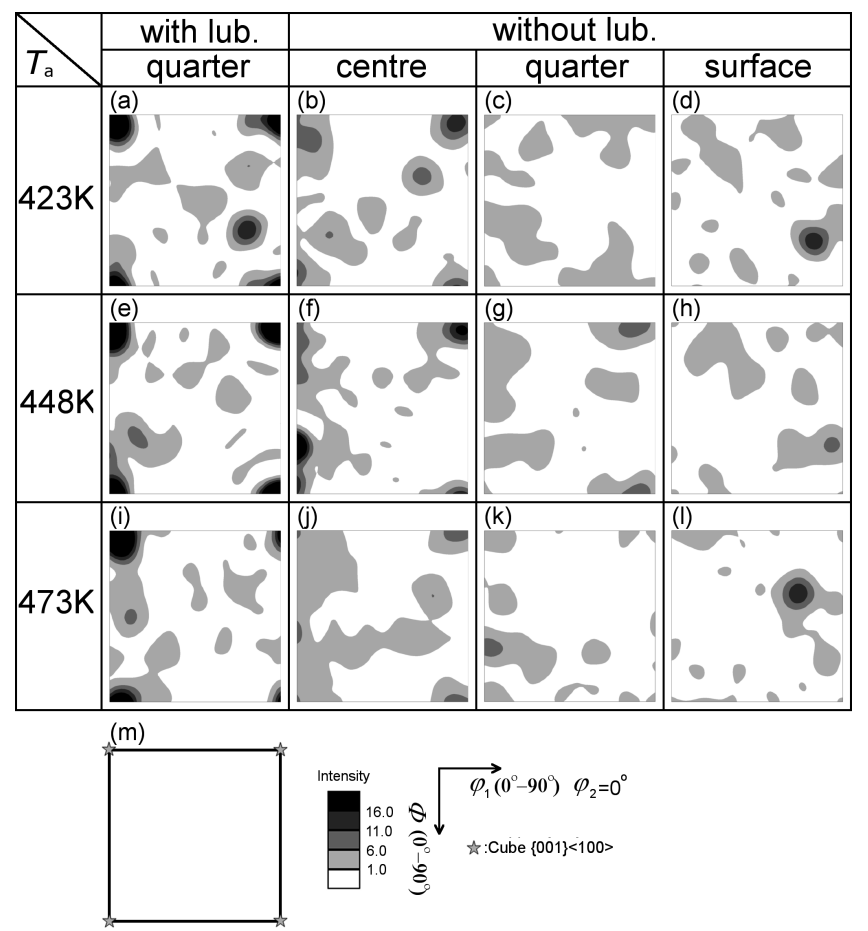

Fig. 11 ODF map on $\phi_{2}=0^{\circ}$ section of ARB processed 4N-Cu: (a)-(d) are ARB $6 \mathrm{c}$ annealed at $423 \mathrm{~K}$, (e)-(h) are ARB $6 \mathrm{c}$ annealed at $448 \mathrm{~K}$ and (i)-(m) are ARB $6 \mathrm{c}$ annealed at $473 \mathrm{~K}$. The first column from the left is the results at quarter of with lubrication. The second, third, and the fourth columns from the left are at centre, quarter, and surface of ARB without lubrication. (m) Cube on $\phi_{2}=0^{\circ}$ section.

bottom rows are the result of annealing temperature at $423 \mathrm{~K}$, $448 \mathrm{~K}$ and $473 \mathrm{~K}$, respectively. The first column from the left is the results of ARB with lubrication. The second, third, and the fourth columns from the left are at centre, quarter, and surface of ARB without lubrication. From those figures, Cube orientation can be seen only for annealed ARB $6 \mathrm{c}$ with lubrication. In contrast, ARB $6 \mathrm{c}$ without lubrication at all the regions show no Cube orientation for all annealing temperatures and rather random texture.

Cube orientation is known as typical rolling-recrystallization of fcc metals having relatively high SFE, and therefore, the present results about annealed ARB with and without lubrication are reasonable. Thus, ARB with lubrication is regarded to be equivalent to the conventional rolling from rolling texture evolution and annealed texture point of views. It should be also pointed out that annealed $4 \mathrm{~N}-\mathrm{Cu}$ ARB processed without lubrication gives random texture. In other words, additional shear strain is useful from the industrial point of view, if strong Cube orientation wanted to be avoided as a result of annealing of fcc metals having relatively high SFE. Because additional shear strain reduces the strength of typical fcc rolling texture, but, the mechanism why the random texture appeared after the annealing of $\mathrm{Cu}$ ARB processed without lubrication is unknown. Thus, the further investigation is requested for revealing the mechanism to appear the random texture.

\section{Conclusions}

The microstructure and texture evolution of ARB pro- 
cessed $4 \mathrm{~N}-\mathrm{Cu}$ with and without lubrication were compared in addition to the mechanical properties. Furthermore, annealing at the temperature range of $423 \mathrm{~K}-473 \mathrm{~K}$ was applied for $4 \mathrm{~N}-\mathrm{Cu} \mathrm{ARB}$ with and without lubrication, and, softening was observed at all temperatures. The followings were confirmed by the experiments and the analysis.

(1) The surface condition of the rolls with and without lubrication affect microstructure and texture evolution of ARB processed $4 \mathrm{~N}-\mathrm{Cu}$ via additional shear strain associated with the friction between the surfaces of rolls and sheets. The grain-subdivision is accelerated in the ARB process without lubrication due to the additional shear strain during rolling.

(2) Since the additional shear strain strongly affects adjacent to the surface, the effect of additional shear strain is more prominent at surface region than that in the inside of the sheets. However, the effect of additional shear strain still exists close to the centre since the centre was the surfaces of the sheet before the last roll bonding.

(3) $45^{\circ}$ rotated Cube around ND was the main component of the texture in $4 \mathrm{~N}-\mathrm{Cu}$ processed by ARB without lubrication, whereas, that produced by ARB with lubrication was Copper orientation. From texture point of view, ARB with lubrication is equivalent to the conventional cold rolling with lubrication.

(4) When annealing was applied for $4 \mathrm{~N}-\mathrm{Cu}$ ARB processed with and without lubrication, Vickers hardness decreases from about $140 \mathrm{Hv}$ down to about $70 \mathrm{Hv}$, and then, saturates. At the saturation region, Cube orientation appeared as the main component in $4 \mathrm{~N}-\mathrm{Cu} \mathrm{ARB}$ processed with lubrication, whereas, no specific texture appears in $4 \mathrm{~N}$ $\mathrm{Cu} \mathrm{ARB}$ processed without lubrication. From application point of view, $45^{\circ}$ rotated Cube around ND formed by additional shear strain can be used for the texture control of sheets when annealing is applied.

\section{Acknowledgements}

This study was financially supported by a Grand-in-aid for Scientific Research on Innovative Area "Bulk Nanostructured Metals" No. 22102006 through the Ministry of Education, Culture, Sports, Science and Technology (MEXT), Japan. The authors are most grateful to Professor Nobuhiro Tsuji, Kyoto University for the use of rolling mill in his laboratories.

\section{REFERENCES}

1) A. Azushima, R. Kopp, A. Korhonen, D.Y. Yang, F. Micari, G.D. Lahoti, P. Groche, J. Yanagimoto, N. Tsuji, A. Rosochowski and A. Yanagida: CIRP Annu.-Manuf. Technol. 57 (2008) 716-735.

2) S. Onaka: J. Japan Inst. Metals 74 (2010) 165-170.

3) N. Kamikawa, N. Tsuji and Y. Minamino: Sci. Technol. Adv. Mater. 5 (2004) 163-172.

4) N. Kamikawa, N. Tsuji, X.X. Huang and N. Hansen: Mater. Trans. 48 (2007) 1978-1985.

5) N. Kamikawa, T. Sakai and N. Tsuji: Acta Mater. 55 (2007) 5873-5888.

6) T. Inoue and N. Tsuji: Comput. Mater. Sci. 46 (2009) 261-266.

7) J. Hirsch and K. Lucke: Acta Metall. Mater. 36 (1988) 2863-2882.

8) J. Hirsch and K. Lucke: Acta Metall. Mater. 36 (1988) 2883-2904.

9) C.P. Heason and P.B. Prangnell: Mater Sci Forum. 408-4 (2002) 733738.

10) S.Y. Li, F.W. Sun and H. Li: Acta Mater. 58 (2010) 1317-1331.

11) K. Kashihara, W. Ikushima, Y. Miyajima, D. Terada and N. Tsuji: Mater. Trans. 52 (2011) 825-829.

12) P.P. Bhattacharjee, Y. Takatsuji, Y. Miyajima, D. Terada and N. Tsuji: Metall. Mater. Trans., A Phys. Metall. Mater. Sci. 43A (2012) 24422452.

13) Y. Miyajima, D. Kashioka and N. Tsuji: Mater. Sci. Forum 702-703 (2012) 173-176.

14) Y.B. Zhang, O.V. Mishin and A. Godfrey: J. Mater. Sci. 49 (2014) 287293.

15) P.L. Sun, W.J. Li and W.C. Hsu: J. Mater. Sci. 51 (2016) 3607-3618.

16) N. Takata, K. Yamada, K. Ikeda, F. Yoshida, H. Nakashima and N. Tsuji: Mater. Trans. 48 (2007) 2043-2048.

17) N. Tsuji: Tetsu-to-Hagane 94 (2008) 582-589. 